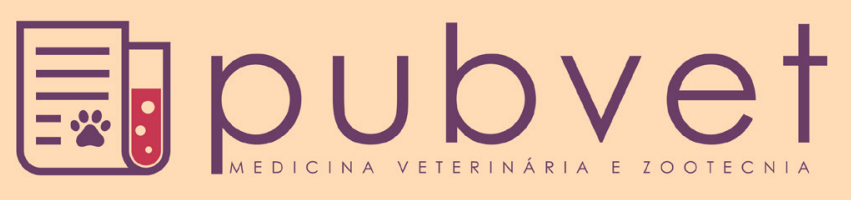

HTTP://DX.DOI.ORG/10.22256/PUBVET.V11N6.554-560

\title{
Manejo pré-abate, bem-estar e suas relações com a qualidade da carne ovina: Revisão
}

\author{
Aparecida de Fátima Madella-Oliveira ${ }^{1 *}$, Celia Raquel Quirino \\ ${ }^{1}$ Professora do Instituto Federal do Espírito Santo - Campus de Alegre, Alegre, ES, Brasil. amadella@ifes.edu.br \\ ${ }^{2}$ Professora da Universidade Estadual do Norte Fluminense - UENF, Campos dos Goytacazes, RJ. Brasil. crq@uenf.br \\ *Autor para Correspondência
}

RESUMO. Esta revisão tem por objetivo abordar de forma sucinta os aspectos relacionados ao bem-estar durante o período de manejo pré-abate dos ovinos com ênfase na qualidade da carne. O manejo pré-abate corresponde à etapa final da criação dos animais, fase que antecede o abate. O manejo pré-abate inclui uma série de atividades, como: tempo de jejum, embarque, transporte, desembarque, alojamento nas baias do frigorífico, período de descanso, atordoamento e abate que necessitam ser realizados com bem-estar animal. Animais de produção que são manejados na ausência do bem-estar, podem ter a qualidade da carne afetada. Conclui-se que a ovinocultura no Brasil é carente de informações dos benefícios de um manejo com bem-estar. São necessários vários estudos e conscientização na cadeia produtiva de ovinos, relacionados com o comportamento e bem-estar animal.

Palavras chave: Comportamento animal, ovinocultura, transporte

\section{Pre-slaughter management, welfare and its relationships with the meat quality of ovine: Review}

\begin{abstract}
This review aims to address briefly the aspects related to welfare during preslaughter sheep with emphasis on meat quality management. The pre-slaughter handling is the final step in creating the animals, phase before slaughter. The pre-slaughter management includes a number of activities such as: time of fasting, loading, transportation, unloading, accommodation bays in the refrigerator, rest period, stunning and slaughter that need to be performed with animal welfare. Livestock that are handled in the absence of well-being may have affected the quality of the meat. We conclude that the sheep industry in Brazil is lacking information of the benefits of a management with wellness. Several studies and awareness in the production chain of sheep-related behavior and animal welfare are needed.
\end{abstract}

Keywords: Animal behaviour, sheep breeding, transport

\section{Manejo pre sacrificio, bienestar y sus relaciones con la calidad de la carne ovina: Revisión}

RESUMEN. Esta revisión tiene como objetivo abordar de forma sucinta los aspectos relacionados al bien estar durante el período de manejo antes del sacrificio de los ovinos con énfasis en la calidad de la carne. El manejo antes del sacrificio corresponde a la etapa final de la cría de los animales, fase que antecede el sacrificio. El manejo antes del sacrificio incluye una serie de actividades, como: tiempo de yeyuno, embarque, transporte, desembarque, alojamiento en los corrales del frigorífico, período de descanso, aturdimiento y abate que necesitan ser realizados sin sufrimiento del animal. Animales de producción 
que son manejados sin bien estar, pueden tener la calidad de la carne afectada. Se concluí que la producción de ovinos en el Brasil es carente de informaciones de los beneficios de un manejo con bien estar. Son necesarios varios estudios y concientización en la cadena productiva de ovinos, relacionados con el comportamiento y bien estar animal.

Palabras clave: comportamiento animal, producción de ovinos, transporte

\section{Introdução}

A ovinocultura vem despontando como uma importante atividade de sustentabilidade, abrindo perspectivas de consolidação no agronegócio brasileiro superando o precário estágio de subsistência. Contudo, alguns problemas ainda precisam ser superados para que essa criação seja rentável, como por exemplo, o manejo de todas as etapas da criação desses animais, principalmente o manejo pré-abate, onde ocorre o maior número de perdas devido ao estresse ocasionado aos animais, que afeta consequentemente o bem-estar e a produção (Lima and Barbosa Filho, 2013). Uma das principais preocupações dos mercados consumidores é à qualidade da carne, que além do ponto de vista sanitário, é determinado basicamente por maciez, suculência e sabor, que está diretamente ligada ao manejo e ao bem-estar dos animais.

O bem-estar de ovinos tem sido bastante pesquisado ao redor do mundo e, como consequência, tem sido produzido conhecimento capaz de fornecer subsídio para a elaboração de diretrizes para os diversos tipos de produção de ovinos (Freitas et al., 2017). Os autores continuam a relatar que no Brasil ainda não existe legislação específica para ovinos e a quantidade de pesquisas é insuficiente para determinar as necessidades das diferentes raças, nos diversos sistemas de produção e condições climáticas brasileiras (Freitas et al., 2017). A implantação das técnicas de manejo racional podem evitar os danos na carcaça, impedido que os animais no período do manejo pré-abate tenha o aumento do estresse, afetando a qualidade da carne (Colditz et al., 2007).

Dessa forma, serão abordados de forma sucinta os aspectos relacionados ao bem-estar durante o período de manejo pré-abate dos ovinos com ênfase na qualidade da carne.

\section{Bem-estar}

Bem-estar animal é um termo subjetivo, influenciado pelas diferentes interpretações das pessoas e culturas distintas que compõem a sociedade. Há, por isso, um grande debate na comunidade científica a respeito do conceito de bem-estar animal e, principalmente, de sua aplicabilidade aos contextos científico e produtivo. Existem duas correntes principais na forma de avaliar o bem-estar de animais criados em cativeiro. A primeira considera principalmente o estado biológico dos animais em uma dada situação, enquanto a segunda considera principalmente as suas experiências subjetivas (Lima and Barbosa Filho, 2013).

De acordo com (Pinheiro and Brito, 2009), bem-estar animal é o "estado de harmonia entre o animal e seu ambiente, caracterizado por condições físicas e fisiológicas ótimas e alta qualidade de vida do animal". Broom (1991) propõe que bem-estar não é um atributo dado pelo homem aos animais, mas uma qualidade inerente a estes, ou seja, se refere a quanto tem de ser feito para o animal conseguir adaptar-se ao ambiente e ao grau de sucesso com que isto está acontecendo. Um animal está em bom estado de bem-estar se ele está saudável, confortável, bem alimentado, seguro, hábil para expressar seu comportamento normal, e não estiver sofrendo estados desagradáveis de dor, medo ou aflição. $\mathrm{O}$ bemestar animal requer: prevenção de doenças, tratamento veterinário, manejo nutricional e instalações adequadas, e finalmente um abate ou eutanásia humanitário.

Em 1993 no Reino Unido, o comitê "Farm Animal Welfare Concil" propôs as chamadas "Cinco liberdades", visando o BEA, que consistem em manter os animais livres de fome e sede; livres de desconforto; livres de dor, injúrias e doenças; livres para expressar seu comportamento natural e livres de medo e estresse. No entanto, podem ocorrer conflitos entre estas cinco liberdades, como por exemplo, a liberdade para o animal manifestar seu comportamento entra em conflito com o estresse causado nas interações sociais.

$\mathrm{O}$ bem-estar pode variar entre muito ruim e muito bom e pode ser medido cientificamente a partir do estado biológico ao qual o animal se encontra e de suas possíveis escolhas (Broom and Molento, 2004). Nesse contexto, produtividade, sucesso reprodutivo, taxa de mortalidade, comportamentos anômalos, severidade de danos 
físicos, atividade adrenal, grau de imunossupressão ou incidência de doenças, são fatores que podem ser medidos para avaliar o grau de bem-estar dos animais (Broom, 1991, Mench, 1993). Broom and Molento (2004) consideram que o sofrimento normalmente está relacionado com o bem-estar, embora a ausência de sofrimento não seja, necessariamente, sinônimo de bem-estar.

\section{Manejo pré-abate}

$\mathrm{O}$ manejo pré-abate corresponde à etapa final da criação dos animais, fase que antecede o abate propriamente dito. É um processo complexo, que dura aproximadamente 24 horas e é considerada a etapa da cadeia produtiva que exerce provavelmente maior influência nos índices de quantidade e qualidade do produto final, causando estresse e comprometendo o bem-estar animal (Apple et al., 2005).

Essa etapa inclui uma série de atividades envolvendo a preparação e o manejo dos animais para o transporte e abate, são eles: tempo de jejum, embarque, transporte, desembarque, alojamento nas baias do frigorífico, período de descanso, atordoamento e abate. Se essas atividades não forem realizadas adequadamente podem ocorrer perdas quantitativas e qualitativas na carne. São muitos os potenciais agentes estressores envolvidos no manejo pré-abate, os quais podem ocasionar alterações fisiológicas e metabólicas decorrentes do desconforto físico e/ou emocional (Dalla Costa et al., 2006). Essas alterações comportamentais influenciam nos produtos e subprodutos dessas espécies, tendo como resultado mudanças de textura, sabor, e por vezes, liberando substâncias produzidas durante o período de estresse originado do manejo pré-abate (Barbosa Filho and Silva, 2004).

\section{Tempo de jejum}

$\mathrm{O}$ animal deve ficar sem dieta hídrica e sem ingerir alimentos por 24 horas e ter um descanso, neste período, em local calmo e ventilado (Dalla Costa et al., 2009, Dalla Costa et al., 2010). O período de jejum pré-abate é adotado para reduzir a contaminação da carcaça com conteúdo do intestino durante o processo de abate. No entanto, se houver restrição prolongada de água, os animais se desidratam, podendo causar no verão perda de peso entorno de $10 \%$, comprometendo também a quantidade e a qualidade da carne (Dalla Costa et al., 2010). O jejum antes do abate reduz a incidência de carne pálida, mole e exsudativa (carne PSE). No entanto, tais ganhos em economia e produto de qualidade devido à privação do alimento podem ser negativos com relação à diminuição no rendimento de carcaças, levando a lesões nas carcaças causadas por brigas, incidência de carne escura, firme e seca (carne DFD), devido ao prolongado estresse pré abate e redução no bem-estar do animal (Dalla Costa et al., 2009, Dalla Costa et al., 2010).

Beattie et al. (2002) concluíram que a privação de alimento por 12 horas pré-abate não afeta a performance, peso da carcaça, qualidade da carne e o bem-estar dos animais. O produtor se beneficia na economia de ração/animal, redução de resíduos e os consumidores se beneficiam com a aquisição de um alimento saudável e seguro. É importante também garantir o fornecimento constante de água aos animais, até o momento do embarque.

\section{Embarque, transporte e desembarque}

Embarque dos animais na fazenda é o início do processo de pré-abate dos animais, pois é o processo em que os animais estarão susceptíveis a entrar em estresse. Em relação ao embarque de animais, o que ocorre na maioria das vezes nesta etapa, é que os responsáveis por embarcar os animais nos caminhões de transporte não têm nenhum conhecimento dos princípios básicos do bem-estar (Miranda-de la Lama et al., 2012). Além disso, utilizam ferrões ou choques elétricos, comprometendo a qualidade da carcaça, que poderá sofrer lesões durante o processo "forçado" de condução e entrada dos animais no caminhão de transporte (Barbosa Filho and Silva, 2004).

O transporte mais utilizado no Brasil para conduzir os animais aos matadouros frigoríficos é o rodoviário. Geralmente ocorrem no transporte, problemas de contusões e lesões traumáticas como fraturas e luxações, resultantes da imprudência do condutor, pelas más condições de acesso, pela falta de conservação das estradas, etc. Não raramente, ocorre a morte dos animais, comumente por asfixia. Nessa etapa devem ser minimizadas todas as causas que possam desencadear o estresse dos animais (Hoffman and Lühl, 2012, Jago et al., 1997).

Alguns cuidados são recomendados, como: minimizar o tempo gasto para apartar e embarcar os animais, piso do veículo apropriado, não superlotar o veículo, não viajar mais que 36 horas sem fornecer alimento e água aos animais, preferir viajar nas horas frescas do dia ou à noite (Hoffman and Lühl, 2012, Jago et al., 1997, Tarrant et al., 1992). Os caminhões devem ser fechado para 
impedir que os animais possam estar visualizado as imagens do trajeto, outros veículos e edificações, que provocam o aumento do estresse. É importante que a iluminação no interior do veículo seja boa e que não existam sombras, porque as ovelhas precisam ser capazes de ver área em que elas estão se movendo claramente.

Segundo Jago et al. (1997), Tarrant et al. (1992) o transporte representou a segunda maior causa de lesões em carcaças, devido à alta densidade de carga, associada com maior reação de estresse, risco de contusão e números de quedas. Portanto o número de animais a ser transportado deve ser considerado, já que uma super ou sublotação irão causar problemas de lesões e quedas durante o transporte. Miranda-de la Lama et al. (2012), Miranda-de la Lama et al. (2014) descrevem que lotações exageradas produzem hematomas, arranhões, fraturas ósseas, mortes e estresse. Superlotação resulta em escoriações e lesões corporais produzidas por choques com a carroceria e aumenta os custos. Em todos os casos, compromete a qualidade da carne, o rendimento industrial e a lucratividade. A quantidade de espaço necessário, por cada animal dependerá dos seguintes fatores:

1) O peso corporal: Obviamente, quanto maior o animal mais espaço ele necessita, portanto, Knowles (1998) recomendam que as densidades devem ser definidas em termos de $\mathrm{m} 2 / 100 \mathrm{~kg}$ em vez de m2/animal (Knowles, 1998).

2) Presença de lã e da espessura do velo: Estas podem fazer uma grande diferença para a quantidade de espaço necessário e os animais não aparados tem necessidade de maior espaço que os tosados (Knowles, 1998). Grandin (2014) sugere que as ovelhas lanadas têm necessidade de $25 \%$ mais espaços que as ovelhas tosquiadas.

3) Temperatura: Uma densidade de ocupação elevada pode piorar os efeitos das altas temperaturas(Knowles, 1998).

4) Comportamento dos animais durante o transporte: Ovinos tendem a deitar-se menos que suínos durante o transporte, mesmo que tenham espaço suficiente para fazê-lo (Bradshaw et al., 1999).

As operações de embarque e desembarque dos animais, se bem conduzidas, não produzem reações estressantes importantes (Kenny and Tarrant, 1987). O ideal é que os currais de espera sejam no mesmo nível que o piso dos caminhões, eliminando as rampas, mas quando isso não for possível o ângulo formado pela rampa de acesso ao veículo em relação ao solo não deve ser superior a $20^{\circ}$, sendo desejável um ângulo de $15^{\circ}$ (Cortesi, 1994).

Os ovinos, não se deitam imediatamente após o início de uma viagem, mas sim nas primeiras quatro a dez horas (Knowles, 1998, Knowles, 1995), desde que tenham espaço suficiente (Knowles, 1998). Portanto, ovinos não deitam necessariamente durante percursos curtos, mas durante aqueles longos (Grandin, 2014), Sugerese que para as viagens com mais de quatro horas, todos os animais deverão ser capazes de se deitar. Para viagens com duração superior a 12 horas, os animais têm que receber água e comida durante a viagem. Com trajetos mais longos, há uma perda progressiva de peso corporal e de carcaça (Knowles, 1998). A perda de peso vivo tem sido relatada em 5,5-6\%, após 15 horas de transporte (Broom et al., 1996, Knowles, 1995) e em 7-8\% após 24 horas de transporte (Knowles, 1995). A maior parte das perdas ocorreu durante as primeiras 15 horas (Knowles, 1998). Knowles (1995) não encontraram nenhuma evidência de desidratação durante viagens de até 24 horas em temperaturas acima de $20^{\circ} \mathrm{C}$. No entanto, quando temperaturas subiam acima de $20^{\circ} \mathrm{C}$ em uma grande parte da à viagem, havia indicações claras de que os animais ficaram desidratados (Knowles, 1998).

\section{Periodo de descanso e alojamento nas baias}

Os períodos de descanso de longas viagens são considerados como um meio de prevenir os efeitos de privação alimentar e de água, mas, segundo o Comitê Científico de Saúde e Bem-estar Animal da União Europeia (2002), períodos descanso curtos, de uma hora, por exemplo são insuficientes e podem até ter efeitos negativos sobe o bem-estar. Em viagens longas são necessários um descanso superior a 24 horas para recuperação do estresse e da desidratação (Knowles, 1998).

Nos alojamentos nas baias no frigorífico é importante que todos os animais tenham acesso a água simultaneamente, para não haver coerência entre os animais e os indivíduos mais fortes não excluírem os mais fracos (Broom and Molento, 2004). O ideal é $30 \mathrm{~cm}$ lineares de bebedouro para cada ovino de $20 \mathrm{~kg}$ e cerca de $30 \mathrm{~cm}$ para os ovinos de $30 \mathrm{~kg}$.

O tempo ótimo de descanso no frigorífico é de 3 horas após o transporte para os animais 
acalmarem e recuperarem do estresse provocado pela viagem (Dalla Costa et al., 2006).

\section{Insensibilização ou atordoamento e abate}

A insensibilização ou atordoamento é uma das operações mais importantes no abate dos animais, o estado de insensibilidade mantém as funções vitais até a sangria (Instrução Normativa $\mathrm{N}^{\circ} 03$, 2000).

Um bom método utilizado é a insensibilização elétrica, que consiste em fazer passar pelo cérebro (tálamo e córtex) uma corrente elétrica alternada e de baixa voltagem (70 a $90 \mathrm{v})$, com intensidade não inferior a 25 miliamperes e por tempo suficiente (Raj, 2000). Esse método evita o sofrimento do animal e o estresse que prejudica a carne deixando-a dura e com sabor alterado. A qualidade da carne está diretamente relacionada com o grau de estresse do animal no momento do abate (Guerrero et al., 2013).

\section{Manejo pré-abate, bem-estar e qualidade da carne}

Os fatores pré-abate podem influenciar na cor, capacidade de retenção de água, $\mathrm{pH}$, entre outros. Contudo, podem afetar também pelas mudanças que ocorrem durante a conversão do músculo em carne, a maciez, suculência, aroma, perdas por cocção, entre outros. Assim, é possível obter carne de melhor qualidade pelo controle do estresse durante a vida do animal até o momento pré-abate (Gottardo et al., 2004).

Animais em estresse desencadeiam uma serie de reações, que vão desde aumento da temperatura corporal e da frequência respiratória, passando por alta concentração de cortisol sérico, glicólise rápida com queda do $\mathrm{pH}$ muscular, rápida desnaturação proteica, resultando em um rápido estabelecimento do rigor mortis. A combinação desses fatores promove alteração na conversão normal do músculo em carne, ficando a carne mais dura e mais escura.

Segundo Renerre (2004), as carnes com uma coloração escura, além de apresentar $\mathrm{pH}$ inadequado, têm efeitos sobre a qualidade e na vida útil deste produto. Já o estresse agudo leva a queda excessiva do $\mathrm{pH}$ e a formação da carne PSE, que não é muito comum para ovinos, e sim para suínos.

Além disso, o pH final do músculo exerce influencia sobre vários aspectos na qualidade da carne, como capacitação de retenção de água
(CRA), perda total de cozimento (PAC) e força de cisalhamento (Rivaroli et al., 2016, Eiras et al., 2016) bem como as propriedades organolépticas: maciez, suculência, flavour, aroma e cor (Campo et al., 2006).

\section{Considerações finais}

A ovinocultura no Brasil é carente de informações dos benefícios de um manejo com bem-estar. São necessários vários estudos e conscientização na cadeia produtiva de ovinos, relacionados com o comportamento e bem-estar animal.

\section{Referências bibliográficas}

Apple, J. K., Kegley, E. B., Galloway, D. L., Wistuba, T. J. \& Rakes, L. K. 2005. Duration of restraint and isolation stress as a model to study the dark-cutting condition in cattle. Journal of Animal Science, 83, 1202-1214.

Barbosa Filho, J. A. D. \& Silva, I. J. O. 2004. Abate humanitário: ponto fundamental do bem-estar animal. Revista Nacional da Carne, $328,36-44$.

Beattie, V. E., Burrows, M. S., Moss, B. W. \& Weatherup, R. N. 2002. The effect of food deprivation prior to slaughter on performance, behaviour and meat quality. Meat Science, 62, 413-418.

Bradshaw, R. H., Randall, J. M., Forsling, M. L., Rodway, R., Goode, J. A., Brown, S. N. \& Broom, D. M. 1999. Travel sickness and meat quality in pigs. Animal Welfare, 8, 3-14.

Broom, D. M. 1991. Animal welfare: concepts and measurement. Journal of Animal Science, 69, 4167-4175.

Broom, D. M., Goode, J. A., Hall, S. J. G., Lloyd, D. M. \& Parrott, R. F. 1996. Hormonal and physiological effects of a 15 hour road journey in sheep: comparison with the responses to loading, handling and penning in the absence of transport. British Veterinary Journal, 152, 593-604.

Broom, D. M. \& Molento, C. F. M. 2004. Animal welfare: concept and related issues-review. Archives of Veterinary Science, 9, 1-11.

Campo, M. M., Nute, G. R., Hughes, S. I., Enser, M., Wood, J. D. \& Richardson, R. I. 2006. Flavour perception of oxidation in beef. Meat Science, 72, 303-311. 
Colditz, I., Ferguson, D., Greenwood, P., Doogan, V., Petherick, J. \& Kilgour, R. 2007. Regrouping unfamiliar animals in the weeks prior to slaughter has few effects on physiology and meat quality in Bos taurus feedlot steers. Animal Production Science, 47, 763-769.

Cortesi, M. L. 1994. Slaughterhouses and humane treatment. Revue Scientifique et Tecnnique Office International des Epizooties, 13, 171193.

Dalla Costa, O. A., Coldebella, A., Costa, M. J. R. P., Faucitano, L., Peloso, J. V., Ludke, J. V. \& Scheuermann, G. N. 2006. Período de descanso dos suínos no frigorífico e seu impacto na perda de peso corporal e em características do estômago. Ciência Rural, 36, 1582-1588.

Dalla Costa, O. A., Ludke, J. V., Coldebella, A., Kich, J. D., Costa, M. J. R. P., Faucitano, L., Peloso, J. V. \& Dalla Roza, D. 2009. Efeito do manejo pré-abate sobre alguns parâmetros fisiológicos em fêmeas suínas pesadas. Ciência Rural, 39, 852-858.

Dalla Costa, O. A., Ludke, J. V., Costa, M. J. R. P., Faucitano, L., Peloso, J. V. \& Dalla Roza, D. 2010. Efeito das condições pré-abate sobre a qualidade da carne de suínos pesados. Archivos de Zootecnia, 59, 391-402.

Eiras, C. E., Ornaghi, M. G., Valero, M. V., Rivaroli, D. C., Guerrero, A. \& Prado, I. N. 2016. How does the dietary cottonseed hull affect the carcass characteristics and meat quality of young bulls finished in a highconcentrate diet? Acta Scientiarum. Animal Sciences, 38, 301-310.

Freitas, A. C. B., Quirino, C. R. \& Bastos, R. 2017. Bem-estar de ovinos: Revisão. PUBVET, 11, 18-29.

Gottardo, F., Ricci, R., Preciso, S., Ravarotto, L. \& Cozzi, G. 2004. Effect of the manger space on welfare and meat quality of beef cattle. Livestock Production Science, 89, 277-285.

Grandin, T. 2014. Animal welfare and society concerns finding the missing link. Meat Science, 98, 461-469.

Guerrero, A., Valero, M. V., Campo, M. M. \& Sañudo, C. 2013. Some factors that affect ruminant meat quality: from the farm to the fork. Review. Acta Scientiarum. Animal Sciences, 35, 335-347.
Hoffman, L. C. \& Lühl, J. 2012. Causes of cattle bruising during handling and transport in Namibia. Meat Science, 92, 115-124.

Jago, J. G., Harcourt, R. G. \& Matthews, L. R. 1997. The effect of road-type and distance transported on behaviour, physiology and carcass quality of farmed red deer (Cervus elaphus). Applied Animal Behaviour Science, 51, 129-141.

Kenny, F. J. \& Tarrant, P. V. 1987. The behaviour of young Friesian bulls during social regrouping at an abattoir. Influence of an overhead electrified wire grid. Applied Animal Behaviour Science, 18, 233-246.

Knowles, T. G. 1998. A review of the road transport of slaughter sheep. Veterinary Record, 143, 212-219.

Knowles, T. G. A.r. o. p.t. m. a. y. c. V. R., v.137, p.406-407. 1995. A review of post transport mortality among younger calves. Veterinary Record, 137, 406-407.

Lima, L. R. \& Barbosa Filho, J. A. D. 2013. Impacto do manejo pré-abate no bem-estar de caprinos e ovinos. Journal of Animal Behavior Biometeorology, 1, 52-60.

Mench, J. A. 1993. Assessing animal welfare: An overview. Journal of Agricultural \& Environmental Ethics, 6, 68-75.

Miranda-de la Lama, G. C., Villarroel, M. \& María, G. A. 2014. Livestock transport from the perspective of the pre-slaughter logistic chain: a review. Meat Science, 98, 9-20.

Miranda-de la Lama, G. C., Salazar-Sotelo, M. I., Pérez-Linares, C., Figueroa-Saavedra, F., Villarroel, M., Sañudo, C. \& Maria, G. A. 2012. Effects of two transport systems on lamb welfare and meat quality. Meat Science, 92, 554-561.

Pinheiro, A. A. \& Brito, F. I. 2009. Bem-estar e produção animal. EMBRAPA, Sobral.

Raj, M. 2000. Efeito dos métodos de atordoamento e de abate sobre a qualidade da carne de porco. 1 Conferência Internacional Virtual sobre Qualidade de Carne Suina. Concórdia.

Renerre, M. 2004. La couleur de la viande et sa mesure. INRA, 1, 63-68.

Rivaroli, D. C., Guerrero, A., Valero, M. M., Zawadzki, F., Eiras, C. E., Campo, M. M., 
Sañudo, C., Jorge, A. M. \& Prado, I. N. 2016. Effect of essential oils on meat and fat qualities of crossbred young bulls finished in feedlots. Meat Science, 121, 278-284.

Tarrant, P. V., Kenny, F. J., Harrington, D. \& Murphy, M. 1992. Long distance transportation of steers to slaughter: effect of stocking density on physiology, behaviour and carcass quality. Livestock Production Science, $30,223-238$.

\section{Article History:}

Received 15 February 2017

Accepted 4 April 2017

Available on line13 May 2017

License information: This is an open-access article distributed under the terms of the Creative Commons Attribution License 4.0, which permits unrestricted use, distribution, and reproduction in any medium, provided the original work is properly cited. 